\section{Agouti-related peptide 2 facilitates convergent evolution of stripe patterns across cichlid fish radiations}

\author{
Claudius F. Kratochwil ${ }^{1,2,3 *}$, Yipeng Liang ${ }^{1}$, Jan Gerwin ${ }^{1,3}$, Joost M. Woltering ${ }^{1}$, \\ Sabine Urban ${ }^{1,3}$, Frederico Henning ${ }^{1,4}$, Gonzalo Machado-Schiaffino ${ }^{1,5}$, \\ C. Darrin Hulsey ${ }^{1,3}$, Axel Meyer ${ }^{1,3 *}$
}

The color patterns of African cichlid fishes provide notable examples of phenotypic convergence. Across the more than 1200 East African rift lake species, melanic horizontal stripes have evolved numerous times. We discovered that regulatory changes of the gene agouti-related peptide 2 (agrp2) act as molecular switches controlling this evolutionarily labile phenotype. Reduced agrp2 expression is convergently associated with the presence of stripe patterns across species flocks. However, cis-regulatory mutations are not predictive of stripes across radiations, suggesting independent regulatory mechanisms. Genetic mapping confirms the link between the agrp2 locus and stripe patterns. The crucial role of agrp2 is further supported by a CRISPR-Cas9 knockout that reconstitutes stripes in a nonstriped cichlid. Thus, we unveil how a single gene affects the convergent evolution of a complex color pattern.

S tephen Jay Gould famously posited that if it were possible to rerun the "tape of life," outcomes would be different (1). The relative importance of determinism and contingency during evolution is still far from settled $(2,3)$. But for particular groups of organisms, one can now test Gould's hypothesis. For instance, in less than 8 million to 12 million years, more than 1200 species of cichlid fishes have evolved to form repeated adaptive radiations in the East African Rift Valley lakes, such as Lakes Victoria, Tanganyika, and Malawi (Fig. 1B) (4-8). These adaptive radiations have given rise to a large diversity of species displaying various color patterns (Fig. 1, C to N), including the repeated occurrence of melanic horizontal stripes (Fig. 1A and supplementary text). Convergent evolution is prevalent in the East African cichlid radiations (9-11), providing a replicated natural experiment whereby distantly related species from independent adaptive radiations can be used to determine what mechanisms have generated these recurrent phenotypes (12-16). More specifically, we address whether horizontal stripes, a convergent phenotype, have an identical, similar, or different molecular bases across the independent adaptive radiations of cichlid fishes.

Previously (17) - using a genetic mapping panel of two Lake Victoria species, Pundamilia nyererei

1Department of Biology, University of Konstanz, Konstanz, Germany. 'Zukunftskolleg, University of Konstanz, Konstanz, Germany. International Max Planck Research School for Organismal Biology (IMPRS-OB), Max Planck Institute for Ornithology, Konstanz, Germany. ${ }^{4}$ Department of Genetics, Institute of Biology, Federal University of Rio de Janeiro (UFRJ), Rio de Janeiro, Brazil. ${ }^{5}$ Department of Functional Biology, Area of Genetics, University of Oviedo, Oviedo, Spain.

*Corresponding author. Email: claudius.kratochwil@ uni-konstanz.de (C.F.K.); axel.meyer@uni-konstanz.de (A.M.)
(Pnye, nonstriped, Fig. 1E) and Haplochromis sauvagei (Hsau, striped, Fig. 1C)-we found that horizontal stripes (Fig. 2C) are inherited as a some 18 (Fig. 2A). This was confirmed by a second cross involving the same nonstriped species and another striped species, $H$. chilotes (Hchi, striped) (Fig. 2A and supplementary text). To more precisely isolate the causal genetic interval for stripe presence, we fine-mapped the trait using recombinant $\mathrm{F}_{2}$ individuals of the Pnye $\times$ Hsau cross and reduced the causal interval from 600 to $25 \mathrm{~kb}$ (fig. S1). This interval contained the genes agouti-related peptide 2 (agrp2), v-type proton ATPase subunit d 2 (atp6VOd2), and an unknown gene (unk) (Fig. 2B). The resequencing of all coding regions revealed no fixed missense or nonsense mutations (fig. S2), suggesting that cis-regulatory variation determines stripe presence.

The teleost-specific agrp2 (fig. S3) is a strong candidate gene for stripes because its paralogs have been previously associated with pigmentation phenotypes (18-20). To test for agrp2 expression differences between nonstriped (Pnye) and striped (Hsau) Lake Victoria cichlids, we performed quantitative polymerase chain reaction (qPCR; Fig. 2D and fig. S5) on a number of adult tissues, including skin (supplementary text). Here, agrp2 showed a significantly higher expression in the skin of Pnye (Fig. 2D and fig. S4). The lack of consistent expression variation between melanic and nonmelanic regions and generally across dorsoventral and anterior-posterior positions suggests that agrp2 does not shape pigmentation patterns through local expressionlevel variation but rather acts as a general stripe pattern inhibitor (fig. S6). Whereas qPCR reogs and neighboring genes (supplementary text), recessive Mendelian trait mapping to chromovealed no such expression differences for paral-
qPCRs on $\mathrm{F}_{2}$ Pnye/Hsau hybrid individuals confirmed that expression differences are linked to the agrp2 locus and exhibit an allelic dosage effect as expected for cis-regulatory mutations (fig. S4).

To identify causal mutations affecting both agrp2 expression and stripe phenotype, we sequenced the agrp2 locus in individuals $(n \geq$ 10 individuals per population) from natural populations of the three hybrid-cross species. We screened for alternatively fixed, fully associated variants with the stripe phenotype in pairwise comparisons of each striped species (Hsau or Hchi) versus the nonstriped Pnye. Our analyses indicated that a 1.1-kb interval within the first agrp2 intron (Fig. 2B) that exhibited shared, alternatively fixed alleles is a strong candidate region for a regulatory element controlling agrp2 expression (fig. S7). To test whether this 1.1-kb interval [enhancer of agrp2 in Pnye (Pnye.enh.a)] contains cis-regulatory elements that could influence interspecific differences between striped and nonstriped species, we tested the elements of both species in a green fluorescent protein (GFP) reporter assay in vivo (supplementary text). It showed that Pnye.enh.a efficiently modulates GFP expression [Tukey's honest significant difference (HSD), $P<0.001]$ and is significantly more potent than the homologous sequence of the striped species Hsau (Tukey's HSD, $P<0.001$ ) (Fig. 2, E and F, and fig. S8). Together, these results indicate that higher expression of agrp2, and thereby the suppression of stripe patterns, is indeed enhanced by Pnye.enh.a (fig. S9).

Our results reveal agrp2 as a major determinant of stripe presence that might be sufficient to suppress stripe patterns in Pnye. To further test this finding, we used CRISPR-Cas9 genome engineering to manipulate agrp2 and to thereby potentially derepress stripe patterns. Pnye eggs were injected with Cas9 and agrp2 guide RNAs, and we obtained four mutants, all of which had nonsense and frameshift mutations within agrp2 (fig. S10 and table S1). These CRISPR-Cas9 mutants developed a continuous midlateral stripe (Fig. 2H and fig. S10) yet no dorsolateral stripe (supplementary text). Because horizontal stripes were never observed in noninjected Pnye individuals (>100 observations; Fig. 2G), this strongly suggests that although species such as Pnye have no stripes, the genomic and developmental machinery for stripe pattern formation is in place, and stripes can reappear in this nonstriped species by experimental manipulation of agrp 2 .

Next, we tested if the expression levels of agrp 2 and stripe patterns are generally associated across other cichlid species from the repeated species flocks of Lakes Victoria, Malawi, and Tanganyika, suggesting a shared molecular basis for convergent stripe phenotypes. Using qPCR on adult skins of striped and nonstriped species of each of the three major East African cichlid radiations (in total, 24 species; fig. S11), we revealed that nonstriped species commonly had higher agrp2 expression levels than striped species (Fig. 3B). This association was confirmed 


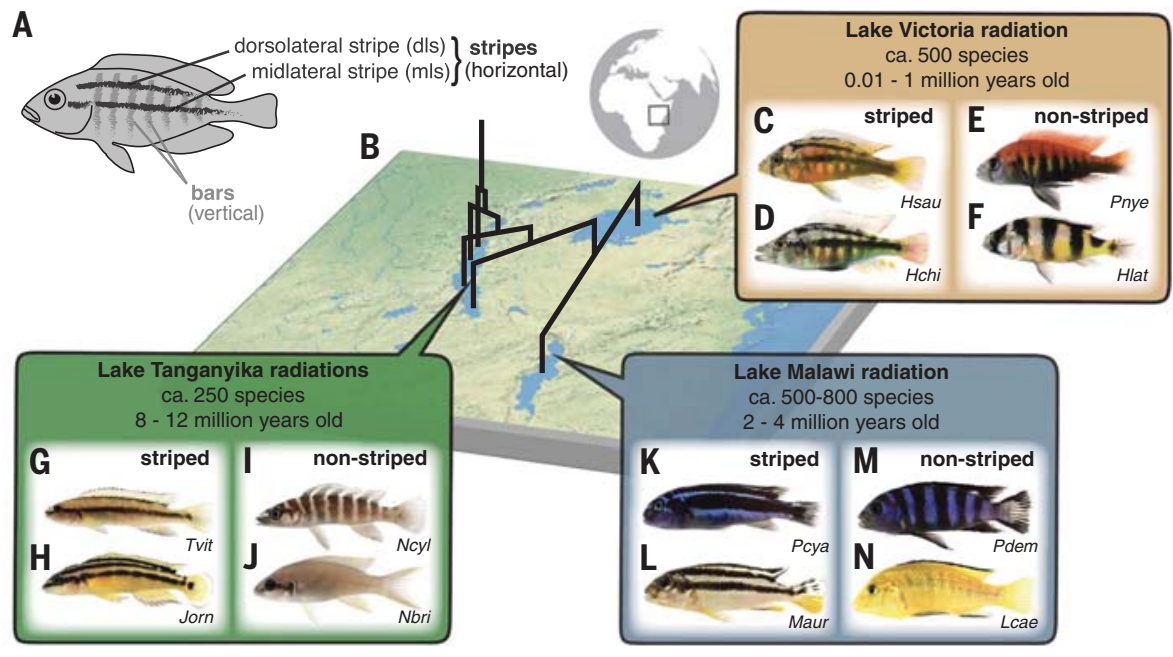

Fig. 1. Convergent evolution of horizontal stripes across African cichlid radiations. (A) Schematic of melanic horizontal stripes and vertical bars. (B) Map of the African Great Lakes Victoria, Tanganyika, and Malawi and superimposed simplified phylogenetic tree of the adaptive radiations of Lakes Tanganyika (green; not all paraphyletic lineages shown), Malawi (blue), and Victoria (orange). (C to $\mathbf{N}$ ) Twelve of the focal striped and nonstriped species of this study, including species from Lakes Victoria $[(C)$ to $(F)]$, Tanganyika $[(G)$ to $(J)]$, and Malawi $[(K)$ to $(N)]$. Hlat, Haplochromis latifasciatus; Jorn, Julidochromis ornatus; Lcae, Labidochromis caeruleus; Maur, Melanochromis auratus; Nbri, Neolamprologus brichardi; Ncyl, Neolamprologus cylindricus; Tvit, Telmatochromis vittatus.
A

B

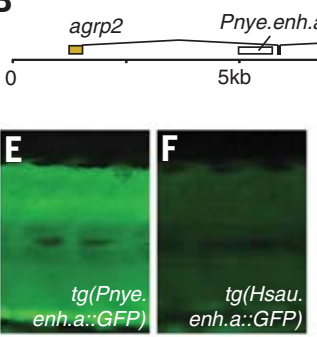

chromosome

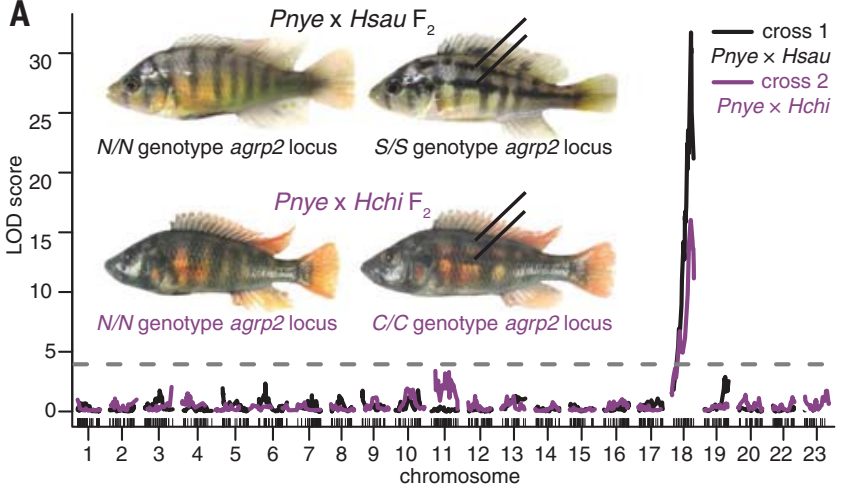

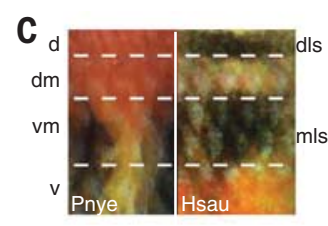

D

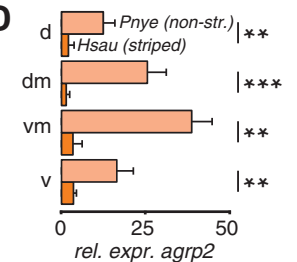

by comparative phylogenetic analyses (Fig. 3A), demonstrating a significant evolutionary association between low agrp2 expression and stripe presence [phylogenetic analysis of variance (ANOVA); mean $P<0.001$; supplementary text].

To determine if this convergence at the phenotypic and agrp 2 gene expression level is also paralleled at the sequence level (16), we comparatively analyzed homologous enh.a sequences across cichlids from Lakes Victoria, Malawi, and Tanganyika. A tree of enh.a revealed substantial sequence variation and resolved striped Lake Victoria species as monophyletic, suggesting a single origin of the striped alleles, whereas striped species of other lakes were not monophyletic (Fig. 3C). None of the nine mutations within enh.a that showed complete association with stripes in Lake Victoria cichlids showed similar stripe association in cichlids of Lakes Malawi or Tanganyika (figs. S12 and S13). Consequently, independent mutations must be affecting agrp 2 expression and thereby stripe patterns across the three major cichlid radiations (Fig. 4D).

Lastly, we tested whether the same locus is responsible for stripe-pattern variation outside of Lake Victoria cichlids using a hybrid cross between the nonstriped Lake Malawi species Pseudotropheus demasoni (Pdem; Fig. 1M) and striped species Ps. cyaneorhabdos (Pcya; Fig. 1K) that also differed in their skin agrp2 expression (fig. S14). We obtained $270 \mathrm{~F}_{2}$ hybrid individuals that were genotyped at the agrp2 locus and phenotyped regarding their stripe patterns (Fig. 4, B and C; fig. S15; and supplementary text). The results revealed significant linkage between the agrp 2 allele and stripe presence (Fisher's exact test, $P=7.6 \times 10^{-8}$; table S2). The allelic variation at the agrp2 locus explains more than $50 \%$ of the phenotypic variance in stripe patterns [Cox-Snell or Nagelkerke pseudo$R^{2}$ from ordered logistic regression; table S3]. Nevertheless, the phenotypic distribution of $\mathrm{F}_{2}$ individuals (49 nonstriped and 221 striped individuals) differed from the Mendelian 3:1 ratio observed in the Lake Victoria crosses (chi-square test, $P<0.001$ ), providing evidence for additional minor modifier loci. These results strongly suggest that agrp2 acts as a major determinant of stripe pattern absence or presence in Lake Malawi (as in the younger Lake Victoria radiation), but additional minor stripe modifiers have evolved or were recruited in the older Lake Malawi radiation (Fig. 4D).

The repeated evolution of horizontal stripes in East African cichlid radiations is facilitated by cis-regulatory evolution of agrp2. Despite its described role and function in the brain (18), we have discovered a hitherto unknown function for this gene in the skin that highlights notable functional similarities between Agrp2 and the mammalian Agouti (Asip) as well as teleost Asip1 (19, 20). From what is known about proteins of the Agouti family, Agrp2 likely acts as an antagonist for the melanocortin receptors Mclr and/or Mc5r (21). Low Agrp2 levels would trigger stripe melanophore proliferation, pigment 

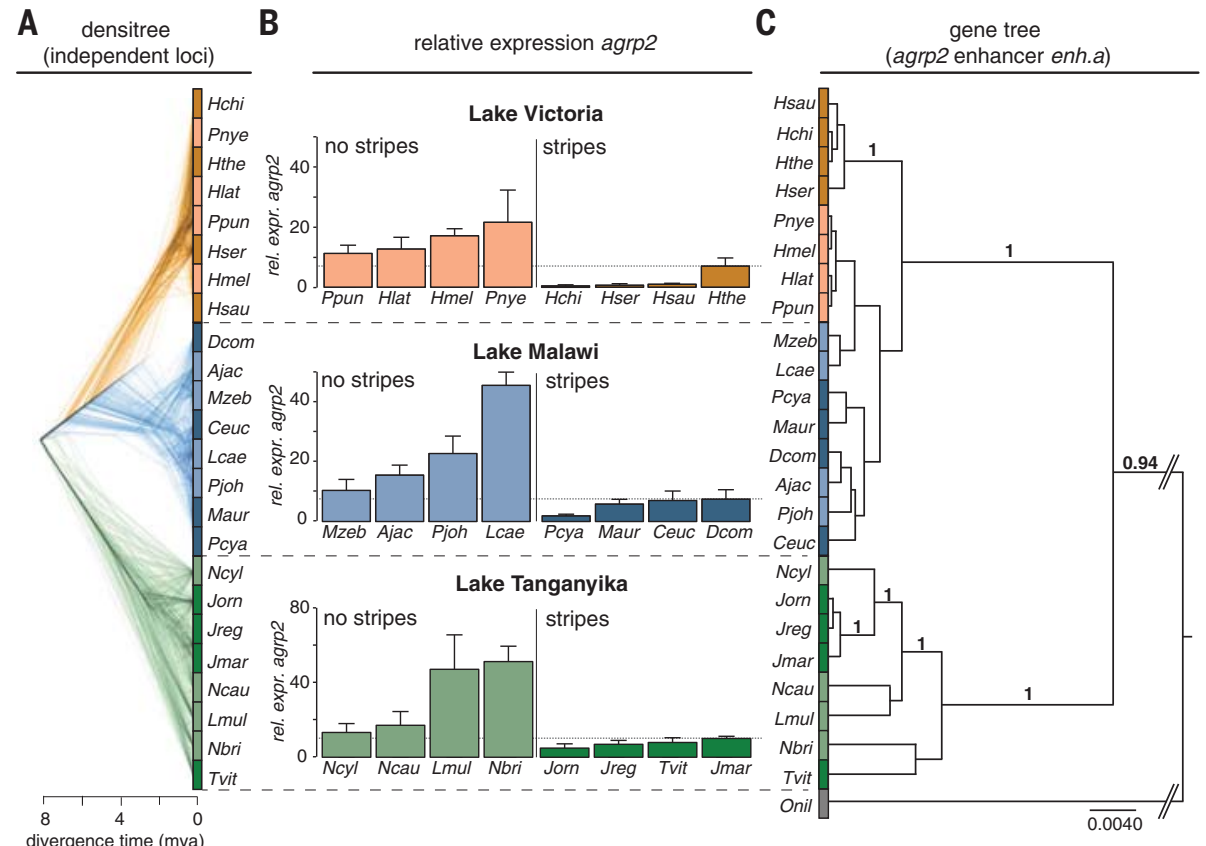

Fig. 3. Regulatory changes of agrp2 are predictive of convergent stripe evolution. (A) Densitree representation of phylogeny for the 24 examined species, including divergence time estimates (8). mya, million years ago. (B) Skin gene expression analysis of agrp2 across adaptive radiations highlights the strong evolutionary association of stripes with low agrp2 expression levels (phylogenetic ANOVA, $P<0.001$ ). Error bars indicate means + SD $(\mathbf{C})$ A gene (locus) tree of the cis-regulatory element enh.a supports a single origin of striped alleles in Lake Victoria. Numbers present posterior probabilities >0.9. Ajac, Aulonocara jacobfreibergi; Ceuc, Cheilochromis euchilus; Dcom, Dimidiochromis compressiceps; Hmel, Haplochromis melanopterus; Hser, Haplochromis serranus; Hthe, Haplochromis thereuterion; Jmar, Julidochromis marlieri; Jreg, Julidochromis regani;

Lmul, Lamprologus multifasciatus; Mzeb, Maylandia zebra; Ncau, Neolamprologus caudopunctatus; Onil, Oreochromis niloticus; Pjoh, Placidochromis johnstoni; Ppun, Pundamilia pundamilia.

A

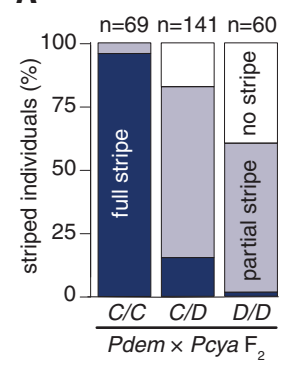

B

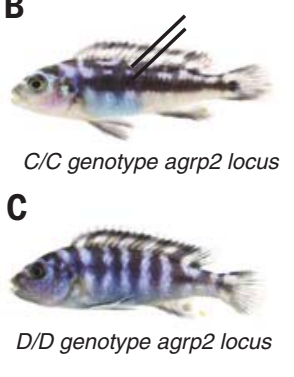

D

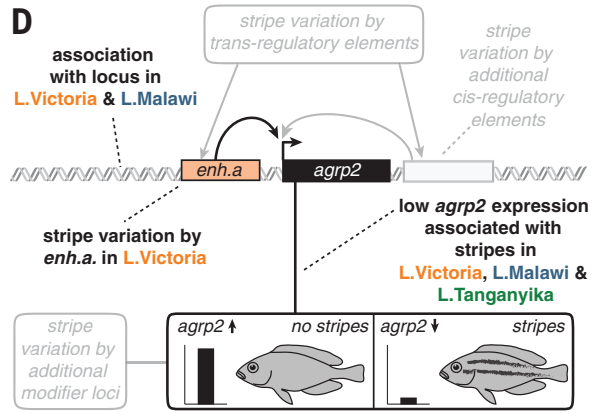

Fig. 4. A shared genomic basis of stripes across cichlid radiations. (A) Stripes are associated with agrp2 alleles in Lake Malawi hybrid $\mathrm{F}_{2}$ individuals (Pdem $\times$ Pcya). Inheritance is not Mendelian, suggesting that additional genetic modifiers exist in Lake Malawi cichlids. (B and $\mathbf{C}$ ) $F_{2}$ hybrids homozygous for the Pcya agrp2 allele (C/C, showing stripes, black lines) and Pdem allele (D/D, no stripes) exhibit clear stripe pattern differences. Parental species are shown in Fig. 1K (striped) and Fig. 1M (nonstriped). (D) Summary of the known (black) and unknown (gray) aspects of the genetic control of cichlid horizontal stripes.

dispersion, and/or pigment production (stripe patterns present), whereas high levels would block these processes (no stripe patterns) (21). Expression levels of agrp2 thereby act as a switch controlling stripe presence and absence. In Lake Victoria, expression-level differences seem to be caused by several mutations in a 1.1-kb intronic regulatory region (enh.a; Fig. 2B) that push the expression of agrp2 levels above or below a threshold that determines the stripe phenotype. Such a threshold-based molecular on-off switch may have permitted the frequent loss as well as reevolution of stripes within East African cichlids. Although the presence of stripes appears to be controlled by differential expression of the same gene (agrp2), causal genetic variants must differ among the independent radiations of Lakes Victoria, Malawi, and Tanganyika (Fig. 4D and figs. S12 and S13). The intermediate phenotypes obtained from the Malawi cross (Fig. 4A), together with the lack of the dorsolateral stripe in the CRISPR-Cas9 mutants (Fig. 2H), provide evidence for additional modifier loci determining stripe presence (Fig. 4D). However, those seem generally less prominent in the young $(<15,000$ years old) Lake Victoria radiation compared to the older ( 2 million to 4 million years old) Lake Malawi radiation (supplementary text).

Regulatory variation of agrp2 provides a molecular basis for the repeated evolution and loss of stripe patterns across cichlid species flocks. Recurrent regulatory evolution at the agrp2 locus constitutes an example of regulatory tinkering $(22,23)$ that might have facilitated the ease and speed of the evolution of both converged and diverged phenotypes that characterizes the East African cichlid radiations. The simplicity of such a threshold mechanism might have permitted the phylogenetically observed rapid losses and reevolutions of stripe patterns. Therefore, Stephen Jay Gould's predictions (I) appear questionable at this evolutionary scale, and if one were to replay the evolution of cichlid adaptive radiations, the results might be surprisingly similar: striped and nonstriped cichlids evolving again and again through regulatory evolution at the agrp2 locus.

\section{REFERENCES AND NOTES}

1. S. J. Gould, Wonderful Life: The Burgess Shale and the Nature of History (W. W. Norton \& Company, 1990).

2. J. B. Losos, T. R. Jackman, A. Larson, K. Oueiroz, L. Rodriguez-Schettino, Science 279, 2115-2118 (1998) 3. J. B. Losos, Improbable Destinies (Penguin, 2017)

4. T. D. Kocher, Nat. Rev. Genet. 5, 288-298 (2004).

5. A. Meyer, T. D. Kocher, P. Basasibwaki, A. C. Wilson, Nature 347, 550-553 (1990)

6. G. F. Turner, Curr. Biol. 17, R827-R831 (2007).

7. M. E. Santos, W. Salzburger, Science $338,619-621$ (2012).

8. D. Brawand et al., Nature 513, 375-381 (2014).

9. O. Seehausen, P. J. Mayhew, J. J. M. Van Alphen, J. Evol. Biol. 12, 514-534 (1999).

10. M. Muschick, A. Indermaur, W. Salzburger, Curr. Biol. 22, 2362-2368 (2012).

11. A. Meyer, Trends Ecol. Evol. 8, 279-284 (1993).

12. S. Kuraku, A. Meyer, Curr. Opin. Genet. Dev. 18, 551-558 (2008).

13. M. E. Protas, N. H. Patel, Annu. Rev. Cell Dev. Biol. 24, 425-446 (2008).

14. R. B. Roberts, J. R. Ser, T. D. Kocher, Science 326, 998-1001 (2009).

15. C. F. Kratochwil, A. Meyer, BioEssays 37, 213-226 (2015).

16. D. L. Stern, Nat. Rev. Genet. 14, 751-764 (2013).

17. F. Henning, H. J. Lee, P. Franchini, A. Meyer, Mol. Ecol. 23 , 5224-5240 (2014)

18. C. Zhang et al., Proc. Natl. Acad. Sci. U.S.A. 107, 20164-20171 (2010)

19. M. Manceau, V. S. Domingues, R. Mallarino, H. E. Hoekstra, Science 331, 1062-1065 (2011).

20. R. M. Ceinos, R. Guillot, R. N. Kelsh, J. M. Cerdá-Reverter, J. Rotllant, Pigment Cell Melanoma Res. 28, 196-209 (2015).

21. L. Cal, P. Suarez-Bregua, J. M. Cerdá-Reverter, I. Braasch, J. Rotllant, Comp. Biochem. Physiol. A Mol. Integr. Physiol. 211 , 26-33 (2017).

22. C. F. Kratochwil, A. Meyer, Curr. Biol. 25, R285-R288 (2015).

23. A. Mazo-Vargas et al., Proc. Natl. Acad. Sci. U.S.A. 114 10701-10706 (2017) 
24. C. F. Kratochwil et al., Data from: Agouti-related peptide 2 facilitates convergent evolution of stripe patterns across cichlid fish radiations. Dryad (2018); doi:10.5061/dryad. h1107nd.

\section{ACKNOWLEDGMENTS}

The authors thank S. Finkernagel for her exceptional technical assistance; A. Kautt, A. Nater, J. Torres-Dowdall, and the anonymous referees for insightful comments that improved the manuscript; and the University of Konstanz bioimaging center and animal facility. Funding: This work was supported by the Swiss National Science Foundation (P2BSP3_148629), the Marie Curie Zukunftskolleg Incoming Fellowship Program (291784), the Baden-Württemberg Foundation, the Deutsche

Forschungsgemeinschaft (DFG, KR 4670/2-1), and the Young Scholar Fund of the University of Konstanz (all to C.F.K.). Y.L. is funded by the China Scholarship Council (CSC). A.M. and C.D.H. are funded by DFG and University of Konstanz. Partial funding came from an ERC Advanced Grant GenAdap (no. 293700) to A.M. Author contributions: C.F.K. conceptualized the study; provided funding; planned and supervised all experiments; collected, analyzed, and visualized all data, if not otherwise indicated; and wrote the manuscript. Y.L. performed qPCRs and in situ hybridization. J.G. and S.U. conducted the Lake Malawi cross and assisted with the sequencing and enhancer assay. J.M.W. designed and generated the CRISPR-Cas9 knockouts and helped with the enhancer assay. F.H. and G.M.-S. provided data for Lake Victoria crosses and contributed to their analysis. C.D.H conducted the phylogenetic analysis and edited the manuscript. A.M. conceptualized the study, provided funding, and revised the manuscript. All authors gave comments on the manuscript and approved the final version. Competing interests: The authors declare no competing interests. Data and materials availability: Additional data have been deposited on Dryad (24) and are available in the supplementary materials. 